\title{
Seeing Self: The CAMPOS Model
}

\author{
Mary Lou de Leon Siantz and Lisceth Brazil-Cruz
}

\begin{abstract}
Building an inclusive community that diversifies the fields of science, technology, engineering, and mathematics (STEM) is a daunting task, all the more so given the low numbers of Latinas and other underrepresented minority groups (URM) who enter academic STEM disciplines. This chapter takes an in-depth look at one of the novel ADVANCE initiatives - the Center for the Advancement of Multicultural Perspectives on Science (CAMPOS). The center fosters sustainable institutional transformation by collaborating with traditional campus committees to recruit, retain, and promote excellent faculty committed to inclusion while also engaging the broader UC Davis STEM community. Its core mission is to support the discovery of knowledge though multicultural perspectives. CAMPOS creates an environment that is diversity-driven, mentorship-grounded, and career-success-focused. It recognizes the barriers that URM STEM scientists endure within academia and seeks to mitigate those barriers, highlighting the accomplishments of CAMPOS faculty scholars and making URM STEM scientists visible role models. The center is committed to transforming STEM by developing the leadership skills needed to sustain institutional transformation in laboratories, departments, and universities locally, nationally, and globally. The CAMPOS model can be replicated at other universities seeking to change the face of STEM.
\end{abstract}

Keywords CAMPOS $\cdot$ STEM $\cdot$ Multiculturalism • Transformative leadership

\author{
M. L. de Leon Siantz ( $\varangle)$ \\ Betty Irene Moore School of Nursing, UC Davis Health System, University of California, Davis, \\ 2315 Stockton Blvd. Sacramento, Davis, CA 95817, USA \\ e-mail: deleonsiantz@ucdavis.edu \\ L. Brazil-Cruz \\ UC Davis ADVANCE Program, University of California, Davis, Davis, CA 95161, USA \\ Institutional Effectiveness, Woodland Community College, 2300 E. Gibson Road, Woodland, CA \\ 95776, USA \\ L. Brazil-Cruz \\ e-mail: lizcruz@ucdavis.edu; lbrazil@yccd.edu
}




\section{Purpose of the CAMPOS Initiative}

CAMPOS was created as a core component of the UCD ADVANCE Program's vision for sustainable institutional transformation in support of women in STEM. At UC Davis, given the demographics of the state, we focused initially on Latina scientists. The Center sought to establish an environment that would inspire excellence, develop leadership, foster success among the faculty scholars selected, and, over time, provide the URM role models missing in STEM. It was designed to showcase talented scientists of color, and benefit from their unique and diverse identities. CAMPOS was built on the premise that multicultural perspectives and interdisciplinary partnerships are essential components of innovative scientific discoveries. By creating an inclusive environment that is diversity-driven, mentorship-grounded, and career-success-focused, CAMPOS embodies and advances UC Davis' commitment to transforming STEM in the twenty-first century.

Given the mandate of the NSF ADVANCE-IT grant program as discussed in the Chapter, 'From Affirmative Action to Inclusion,' CAMPOS leaders felt it was important to create a mechanism that would attract and include a diverse core group of women in science. These women, predominantly Latina, could inspire raciallyand ethnically-diverse students, professionals, and faculty alike to envision themselves working and progressing in STEM programs at UC Davis. As the research in the Chapter, 'Assessing Institutionalized Bias,' highlights, the inability to "see self" in a successful long-term career is a clear deterrent to retaining URM STEM undergraduates, graduate majors, professionals, and faculty.

"Seeing self" in this context means observing successful individuals who represent or reflect one's own social identity. It also means not needing to sacrifice our identity in order to "fit in" and be accepted. Our collective experiences, cultures, and perspectives must not only be accepted, we must also see ourselves as capable of excelling in, and of transforming, the historical traditions of STEM disciplines. "Seeing self" encompasses developing self-efficacy - a belief in one's own ability and in the right to claim a "scientist identity." To promote innovations in STEM, we urgently need institutional change that communicates a diverse and inclusive definition of who is, and can be, a scientist.

A CAMPOS faculty scholar is defined as an exceptional scientist in a STEM discipline. CAMPOS scholars are selected for their transformative thinking, unique perspectives, interdisciplinary approaches, and leadership potential to impact their discipline in profound and enduring ways. Their discoveries, innovations, and technological breakthroughs will contribute to the public good, locally, nationally, and globally. They are role models for future scientists and scholars who share their vision of diversity and inclusion as a key component of academic excellence.

\subsection{CAMPOS Aims}

CAMPOS was envisioned to create and sustain an accessible, inclusive community of research collaborators and mentors committed to diversifying the STEM. At its 
inception, CAMPOS aimed to attract, retain, and help to promote Latina STEM scientists. Consequently, we focused on successfully cultivating the research programs of these scholars in order to increase their research productivity and leadership potential as principal investigators, laboratory managers, and future leaders in academia.

CAMPOS faculty scholar's selection criteria included:

1. Final selection among nominees who are candidates in a department's faculty search

2. Commitment and potential contribution to a diverse community of scholars dedicated to broadening participation in STEM and engaging underserved communities

3. Potential to advocate for diversity and inclusion in STEM

4. Knowledge of barriers that URM scholars experience in STEM.

Our CAMPOS program built upon well-established expectations and principles for academic success. First, we knew that the best predictor of academic success and advancement in STEM disciplines at major research universities are the number of publications that result from a well-funded and long-established program of research (Kraimer et al., 2019). Therefore, we linked clusters of new CAMPOS hires with more-senior mentors and leaders in their disciplines as soon as they began their appointment, irrespective of rank on entry. The linkages helped counter feelings of isolation, build and reinforce self-efficacy in their present and future research potential, and establish a vision for leading a successful program of research in a competitive, research-intensive university with largely homogeneous departments.

Furthermore, we knew that research success currently builds on environments that facilitate networking and mentoring opportunities, so we helped create opportunities for partnerships and innovations with the potential for increased research productivity through team science. The presence of CAMPOS on the UC Davis campus has not only brought together senior scientists to consider the importance of multicultural perspectives but also has helped them experience the potential contributions to scientific discovery and innovation that inclusive environments facilitate. By "multicultural" we don't simply mean racially and ethnically diverse, but also a range of different viewpoints generated by gender, nationality, immigration history, language use, and class status. Diverse, inclusive environments provide the infrastructure needed for sustainable transformation of academic cultures within STEM disciplines and departments. The fact that diversity and inclusion lead to successful team science outcomes has been amply demonstrated through the ability of faculty to garner external funding and to increase publication productivity and reach (Bennett \& Gadlin, 2012; Dutcher \& Rodet, 2018).

Research environments such as those provided by CAMPOS are particularly important to scientists who are women of color, as they experience a double bind of perceived difference and isolation of even greater magnitude than white women in science (Miriti, 2020). Compared to their male counterparts, female STEM faculty members consistently report feelings of greater isolation, reduced opportunity for collaboration, and limited recognition of their current and potential contributions (Bergsieker et al. 2020; Farrell \& McHugh, 2020). This occurs despite 
STEM research breakthroughs that increasingly require collaboration across teams of investigators in multiple disciplines, irrespective of their gender, race, or ethnicity.

Finally, given the paucity of advanced degree-holders among women of color, these scholars often feel more committed and motivated than their colleagues to engage and give back to their communities of origin through outreach, mentorship, and application of their acquired expertise. They are typically more motivated to discover knowledge that will improve the quality of life in their communities of origin (Wallerstein, Calhoun et al., 2019). Thus, we see CAMPOS as a potent model for broadening the transformative impacts of interdisciplinary research centers (Jordan, 2006) that endeavor to meet the needs of vulnerable populations. Community-based partnerships to promote STEM education as well as mentorship through research have proven to be effective strategies.

\subsection{CAMPOS Mission}

Truly innovative research requires creative and novel approaches as well as leaders who commit to sustainable transformation that reflect our current multicultural and global society. The more diverse a team is, the more innovative its methods, findings, and solutions can be (Dutcher \& Rodet, 2018). The mission of CAMPOS is to support the discovery of knowledge by promoting diverse perspectives in science, through an inclusive environment that is diversity-driven, mentorship-grounded, and success-focused. This novel approach focuses on creating a community that values and acknowledges the benefits of diversity while promoting excellence in research. Through the support of diverse faculty, and the increased recruitment and retention of such a faculty, CAMPOS promotes a model for enriching scientific knowledge.

There are both short-term and long-term goals involved. A key short-term goal was to create a robust program of research on the premise that diversifying STEM also entails mentoring younger generations of STEM faculty as well as the next generation of students. Long-term goals include making visible and substantial impacts on STEM innovation through (1) national scientific and technological innovations, (2) contributions to complex global challenges facing human populations, and (3) measurable improvements in the gender, cultural, and ethnic diversity of the STEM workforce throughout the United States.

\subsection{The CAMPOS Model}

Institutional Transformation Enabled by University Leadership Support. The success of initiatives such as CAMPOS begins with a vision for institutional transformation that is committed to multicultural perspectives in STEM. Although this vision is rooted in the history of democratic ideals of equality as well as decades of social science and humanities scholarship on social inequality, it has to be "translated" to 
the academic workplace and widely embraced. Transformation in academic STEM fields has occurred through funded initiatives like the NSF ADVANCE program, modeled largely after corporate diversity efforts. The resources provided by such programs are critical to building the infrastructure needed for diversity and inclusion. Those at the top of the organizational hierarchy must embrace both the vision and a peer-reviewed plan of action. It is through the inspiration, resources, and support of the campus executive leadership, working in partnership with faculty, especially underrepresented faculty, that sustainable change can happen in STEM. The perspectives and participation of underrepresented faculty must be central to all stages of transformation - vision, plan of action, implementation, and evaluation. The support of those in mid-level leadership — deans, department chairs, and senior faculty-is also critical because they are the "connective tissue" between faculty and executive leadership.

Together, these constituencies must commit to promoting racial and gender diversity in science, not just because it is the right thing to do but because multicultural perspectives can lead to better science, including novel scientific breakthroughs. As we have learned from developing CAMPOS, "commitment" must be simultaneously socio-cultural and financial: substantial resources provide incentive, time, and funding to support the kind of faculty engagement that will yield lasting change over time. The goal is a broad-based transformation predicated upon the assumption that diversity and inclusion benefit everyone, not only URM faculty.

Creation and Implementation of a Clear Vision. The initial CAMPOS vision was to attract, then successfully retain and promote, a diverse, collaborative community of tenure-track STEM research faculty at UC Davis. To implement its mission and vision, best practices were informed by the work of the campus's other ADVANCE initiatives. These included: (1) mentorship and networking, (2) policy and practices review, (3) inclusive campus climate, and, (4) Social Sciences Research Initiative, or SSRI. The mentorship and networking initiative established standards for mentoring newly hired women scholars in STEM fields from assistant to full professor at UC Davis, while the policy and practices review initiative worked to establish equitable guidelines for the appointment, retention, and promotion of STEM faculty in line with diversity and inclusion goals. The inclusive campus climate initiative created an institution-wide, faculty-led implicit bias training called Strength Through Equity and Diversity (STEAD) for search committee members involved in faculty recruitment. Finally, the SSRI sought to understand the personal and professional experiences of Latinas in STEM - the details of which are presented in the Chapters, 'Latinx Communities and Academic Trajectories,' and 'Making Visible the Invisible: Studying Latina STEM Scholars'.

Together, these four initiatives provided the strategic scaffolding needed to support the fifth ADVANCE initiative, the Center for the Advancement of Multicultural Perspectives in Science (CAMPOS). As noted earlier, CAMPOS was envisioned as a national prototype for achieving institutional transformation in STEM through collaborative, innovative, funded research projects linking CAMPOS scholars to both the UC Davis STEM community more broadly, as well as diverse communities outside of academia. However, establishing such a vision is but the first step. As 
founding director of CAMPOS, I (Dr. Mary Lou de Leon Siantz), firmly believe, along with business leader Joel Barker (1992), that "vision without action is just a dream," that "action without vision does not just pass the time but is a waste of time," and that "vision and action together can change the world" (Barker, 1993, cited in Ritchie, 1999, p. 1).

Implementing a Vision Must Build on Strategic Planning. Research centers for STEM scholars are built on well-crafted plans that have been carefully vetted by faculty and administrative leaders alike (Kantabutra, 2020). Evaluation, supported by concrete results, is a key component of implementation. Centers build momentum via a mission, a vision, and concrete steps for implementation, tied to ongoing evaluation that measures targeted goals and budgets and verifies outcomes. Thus, my role as founding director was to establish a clear roadmap for CAMPOS, creating both a vision and a clear action plan. Devising a blueprint for action was the first step in the implementation phase of CAMPOS.

The initial challenge that CAMPOS faced was overcoming assumptions that supporting gender and ethnic or racial diversity would "lower the bar" or the standards expected of UC STEM faculty. This is a common myth shared by many academic search committees and STEM scientists in general (Flores et al., 2019). Consequently, the selection process for CAMPOS Faculty Scholars had to counter such beliefs about gender, ethnic, and racial diversity. This process included: (1) the review, selection, and identification of a candidate by a search committee as the department's top choice for a tenure track faculty appointment, (2) a dean's letter of offer to that candidate, (3) a copy of the candidate's CV, and (4) a nomination document by the search committee chair to the CAMPOS review committee describing the candidate's appointment in the target department and why he or she was a good fit for CAMPOS. The CAMPOS review committee then evaluated the candidate for potential appointment as a CAMPOS faculty scholar. It was important to the success of CAMPOS that nominees be the top candidate in their department as determined by the normal protocol of the departmental search committee, because the CAMPOS faculty scholar designation is considered an honor and an important component of the candidate's recruitment package. CAMPOS candidates were always invited to speak with me as founding director or a member of the CAMPOS selection committee when on campus.

The CAMPOS faculty scholar selection committee members were named by me and the Vice Provost for Academic Affairs. Tenured full professors in STEM departments, schools, and programs at UC Davis who also represented gender, ethnic, and racial diversity were invited to join the inaugural committee. The committee reviewed all CAMPOS nominations for their potential fit with the CAMPOS mission and participated in campus interviews when possible. I also met with potential candidates to discuss the prestige of the CAMPOS designation and its added benefits to a faculty appointment at UC Davis. These included summer research support, membership in faculty diversity organizations, the CAMPOS Leadership Institute, and paid membership in the National Association of Faculty Diversity, which provided additional faculty leadership development. 
Once formally established, CAMPOS initiated a new cohort into the center's program each academic year. Despite the range of STEM disciplines that CAMPOS scholars represented, they were assigned to the cohort of the year that they began at UC Davis. Cohort establishment built community, helped orient the faculty scholars to UC Davis, and alerted newcomers to expectations for merit, promotion, and retention.

To enable other UC Davis faculty to participate in CAMPOS activities, the CAMPOS faculty affiliates program was established. This program helped affiliated scholars expand their research networks, and, via these networks, build diversity within STEM and enhance campus engagement with underserved communities. All UC Davis faculty (including Academic Senate and Academic Federation faculty from STEM and non-STEM disciplines) who wished to contribute to the CAMPOS mission were eligible to apply to the CAMPOS Faculty Affiliates Program. Affiliates enjoyed a variety of benefits: membership in a collaborative interdisciplinary community interested in developing new research, teaching, and mentoring methods to support diversity at UC Davis; access to networking and mentoring opportunities; and access to support for conference travel and other professional activities aligned with CAMPOS goals. Priority consideration was given to affiliates who enroll in ADVANCE and CAMPOS professional development opportunities as they arose. These included grant-writing workshops, joining the National Center for Faculty Development \& Diversity (NCFDD) Faculty Success Program, and attending space-limited seminars, roundtables, and UC Davis networking events.

Sustainability. With the commitment of UC Davis executive leadership, STEM faculty, and in collaboration with other ADVANCE initiatives on campus, CAMPOS has become a sustainable outcome of the NSF ADVANCE institutional transformation grant. CAMPOS successfully recruited 28 ethnically and racially diverse STEM scientists across 21 STEM disciplines. CAMPOS also piloted mentoring programs for its new hires that are now being extended to all faculty, and it created tools to develop leadership skills and visibility. CAMPOS is now sponsored by the Provost, while the selection of CAMPOS Faculty Scholars occurs under the leadership of the Vice Provost for Academic Affairs together with the CAMPOS director. Overall, CAMPOS programming is managed by the Associate Vice Chancellor for Academic Diversity and is housed in the Office of the Vice Chancellor for Academic Diversity, Equity, and Inclusion.

The center will continue recruiting a diverse array of scholars who meet the academic standards of university search committees and are appointed as faculty in STEM departments. These scholars will continue to have access to mentoring programs, leadership development opportunities, and outreach activities. Over time, they will educate and mentor undergraduate, graduate students, and new faculty about the benefits of interdisciplinary and multicultural perspectives in STEM. Moreover, by connecting and engaging with historically underserved communities, and by prioritizing research agendas that meet the needs of these communities, CAMPOS scholars will increase the relevance of STEM for everyone. 


\subsection{CAMPOS Programming Components}

Mentorship. A key component of successful faculty and academic leaders is mentorship (Corneille et al., 2019). Mentors provide a range of wisdom and experience through their advice, role modeling, and constructive feedback, all of which facilitate research productivity, appointments to scientific review boards, and nominations for prestigious scientific awards. These elements are critical to successful academic careers in STEM. To ensure that incoming faculty will thrive in new work environments, CAMPOS scholars were offered participation in "LAUNCH" committees during their first year, with the option of renewing though their second year. Such committees are composed of the department chair, a successful senior department faculty member, and other faculty members (in or outside the department) who the scholar deems helpful to their success at UC Davis. The LAUNCH committee advises on committee assignments, consideration of committee appointments, student advising, teaching, and preparation for merit or promotion review. They also advise on broader issues such as how to recognize exceptional work in academia across a broad range of metrics and how to flag their own contributions and achievements to their best advantage.

Network Development. Networks are also keys to success in academia (Xu \& Martin, 2011). The CAMPOS faculty scholars and UC Davis faculty are encouraged to network to learn more about CAMPOS and to identify ways to collaborate in research. "Cafecitos," or coffee breaks, were established to for scholars to meet and communicate with other members of the university. The Cafecitos enabled undergraduate, graduate, doctoral, and postdoctoral students to learn about CAMPOS and possible opportunities for involvement; they also facilitated conversations among faculty about grant prospects involving interdisciplinary and innovative team approaches. Cafecitos provided a space to recognize faculty awards for center participants. Finally, these coffee hours allowed CAMPOS faculty scholars to share their expertise through scientific presentations; these were so successfully we decided to develop the Cafecitos into research colloquia events.

Leadership Development. CAMPOS faculty scholars are chosen for their capacity to advance science in their respective STEM disciplines. However wellprepared these scholars might be, multiple factors persist that prevent underrepresented groups from achieving leadership positions. Faculty of color, whether they have earned doctoral degrees or not, are rarely considered for their leadership positions and leadership potential (Freeman et al., 2019). Therefore, one additional goal of CAMPOS was to build leadership skills and confidence for continued success; as a result, the CAMPOS Leadership Institute was established.

The Institute helped prepare CAMPOS scholars for the opportunities that might arise as their careers unfold - as tenured professors, principal investigators of grants and projects, laboratory directors, research team leaders, department chairs, deans, and even chancellors. The Leadership Institute focused on three core competencies: (1) personal skills needed to successfully lead laboratory teams, and to consider potential leadership opportunities over time; (2) the self-efficacy required to navigate 
the merit review, promotion, and retention process at UC Davis; and (3) the expertise required to communicate matters of science to legislative leaders and external constituencies at both state and federal levels in accessible, jargon-free language. The underlying paradigm embraced multicultural perspectives: traditional approaches to leadership were integrated with Latino, Black, and American Indian approaches in order to strengthen the multicultural roots of the CAMPOS faculty scholars (Bordas, 2012). The institute helped participants recognize the competitive advantage that their multicultural perspectives brought to their own leadership potential (Szymanski et al., 2019).

Understanding Personal Leadership Characteristics. CAMPOS scholars are scientists who lead or will lead laboratory teams to innovation and discovery. The goal is to provide them with the skills needed to become successful laboratory leaders who can (1) distinguish themselves in extremely competitive environments, and (2) can help transform laboratories into innovative, nurturing, collaborative, and peoplecentered research environments that support successful programs of research for everyone involved (Maestre, 2019). The Leadership Institute also seeks to prepare scholars to assume leadership positions in academia as department chairs, deans, provosts, chancellors, presidents, as well as in their professional fields more broadly.

CAMPOS was fortunate in having a budget to support the leadership institute through the NSF ADVANCE institutional transformation grant. The budget covered costs for the leadership coach's travel, per diem, and time devoted to training participants. It also paid for the leadership assessments of each participant. These assessments, described in the next section, reflect the skills of each participant and are tailored to each of them as a result. The cost of university rooms needed during the institute and meals were included in the budget. The leadership institute began with a dinner the evening before to set the tone, prestige, and importance of the Institute. The Chancellor, Provost, and other academic leaders were all present at the dinner. The institute not only nurtured leadership skills, it also brought the cohort of participants together as a group through exercises aimed at team-building as well as leadership skills development. The experience was evaluated very positively by participants.

To assess personal leadership styles, each scholar was asked to complete the Myers Brigg Type Indicator (MBTI), a self-report questionnaire (Myers \& Myers, 2010). The MBTI assesses four personal characteristics: (1) Introversion/Extraversion, (2) Sensing/Intuition, (3) Thinking/Feeling, (4) Judging/Perceiving. Each person is said to have one preferred quality from each category, producing 16 unique types (Sonnino, 2016). The assessment provides CAMPOS faculty scholars with insight into: (1) their own personal characteristics, (2) effective communication skills with persons who are very similar or very different from them, and, (3) leadership strengths that need ongoing development over time.

Each scholar was also asked to complete a "Leadership 360" assessment (Toegel $\&$ Conger, 2003). Outstanding leaders are expected to understand their personal styles not only from their own perception but also through the eyes of their peers, managers, supervisors, and members of their team. External assessments from a broader perspective help each scholar to identify and develop the competencies and self-knowledge needed to achieve personal, professional, and leadership excellence. 
A Leadership 360 assessment focuses on: (1) communication skills, (2) decisionmaking abilities, (3) promoting change and innovation, (4) effective working relationships, (5) leadership skills, (6) coaching skills, (7) use of personal and others' strengths, and (8) team development.

In addition, CAMPOS Faculty Scholars had the opportunity to learn directly from leaders in academia. Faculty holding leadership positions led discussions about their own experiences in navigating their own careers as women in STEM science. These leaders included chancellors, vice chancellors, vice provosts, department chairs, ADVANCE program leaders, STEM business leaders, as well as community leaders (for example the Consul General for the Mexican Embassy in Sacramento, who happened to be a woman). These women had all overcome challenges and become leaders in their respective fields and organizations, having been recognized for their exceptional achievements.

The Leadership Institute also provided advice and discussion about the need for and ability to balance family and work, including but not limited to managing familywork conflicts and work overload. These discussions were important to all participants, particularly those who were or were soon to become young mothers and were new to their academic appointment.

Navigating the Promotion, Tenure, and Merit Review Process. CAMPOS was built on the assumption that diversity and inclusion does not end with appointment of women and faculty of color. This is merely the springboard to launch multicultural perspectives in STEM departments. Newly-appointed scholars of diverse backgrounds need to understand how to traverse obstacles of merit review, promotion, and tenure, whether they were appointed as assistant, associate, or full professors.

Training specific to UC Davis faculty merit reviews was provided by the vice provost for Academic Affairs and the chair of the Academic Senate, as well as faculty from the STEM disciplines who were tenured full professors. Women, especially women of color, continue to face barriers that include academic structures and a culture that have proven difficult to change, a deeply entrenched faculty value system, and ingrained sociocultural norms that define social roles and expectations (Burgess et al., 2012; Pearce et al., 2020). CAMPOS faculty scholars (CFS), learned about what to expect in a merit and promotion review. Dossier preparation was discussed using specific examples. The vice provost for Academic Affairs detailed the effective presentation of a dossier and its contents. She also shared available resources that UC Davis provides in grant and project development. The Faculty Senate chair discussed the Academic Senate review process. Tenured professors talked about the workings of personnel committees and their review processes. It was made clear from the start how candidates could take advantage of assistance and how they needed to fully understand each step in the merit review process, including expectations in each rank. The CFS expressed surprise at the level of detail provided as they entered UC Davis to begin their new appointments. They were not familiar with the sizable investment the university was making to ensure their potential success.

Over time, CAMPOS faculty scholars were given the opportunity to talk with members of the CFS selection committee in their departments, while also taking in advice that their own launch committees provided. CFS produced outstanding merit 
reviews, earned promotions, and achieved tenure based on their research achievements, their teaching portfolios, their university service, and their engagement with programs of diversity and inclusion.

Scientific Communication with Legislators. Continued scientific innovation will require exceptional leaders in academe who reflect the ethnic, racial, and gender diversity of the United States. A need exists, then, to prepare the next generation of STEM scientists not only to advance knowledge but also to inform public policy makers at local, state, and federal levels about their research. Thus, the purpose of the Policy Development component of the Leadership Institute is to inspire racially and ethnically diverse STEM scientists to lead and shape such policy through communicating knowledge about STEM research in their districts. For most academics, the chance to discuss their research with legislators occurs at mid-career or later, if it even occurs at all. Scientists of color are rarely viewed as the experts in their fields (Jemison, 2019), so CAMPOS seeks to provide skills to change this view.

To build expertise in communicating science to policy-makers, the Leadership Institute planned for CAMPOS Faculty Scholars to meet with elected state and federal officials.. Many officials eagerly accepted the invitation to meet new URM STEM and learn about their research. The UC Davis Directors of Federal and State Government Relations prepared the CAMPOS Faculty Scholars for their visits to elected representatives and their staffs.

Scholars a-were first introduced to the legislative process at state and federal levels. Each CFS prepared a personal introduction lasting about 30 seconds, which includes their name, title, key area of expertise, as well as a one-page summary of their research, written in lay terminology, designed be left with the policy-maker or a staffer. The CAMPOS's Founding Director, along with administrative leaders (who may include the chancellor, the vice chancellor, and the provost, as well as the Directors of Federal and State Government Relations), then accompany the scholars to caucuses that support science, either at their State Capital offices in Sacramento or at their U.S. Capitol offices in Washington, D.C. The visits were solely an informational exchange as UC government policy and the National Science Foundation prohibits lobbying.

Community Engagement. A key component of the CAMPOS vision has been community engagement, with the goal of engaging CFS in answering questions that are important to local communities throughout California. An important quality that each scholar brings to UC Davis is their commitment to partner with local communities of color, whether through mentorship of students or pursuing research that answers questions of mutual concern. To this end, CAMPOS scientists and faculty are working to engage children and parents K-2 in STEM science, allied with organizations like 4H Clubs, Cesar Chavez Science Day, and summer STEM programs for underrepresented racial and ethnic minority high school students from communities around Northern California.

Building on their participation and success in the CAMPOS initiatives, Scholars are also teaching, mentoring, and inspiring college undergraduates to pursue graduate school in STEM fields. For example, six undergraduate research assistants in the Social Science research initiative applied to graduate school in STEM or Health 
Science as a result of their participation in faculty research through CAMPOS. During their occasional visits to campus, community organizations seek faculty to share their academic journeys. CFS and affiliated faculty are working jointly to establish community-based research to promote environmental changes that affect community health with a team science approach.

Promoting Team Science. One of the key strategic goals CAMPOS has been to revolutionize STEM science as well as the pipeline to STEM fields through "team science." The term can be defined as a "collaborative effort to address scientific challenges leveraging the strengths and expertise of professionals trained in different fields" (Bennett \& Gadlin, 2012). Key elements of a successful team science approach include: (1) a shared vision; (2) development of a shared vocabulary among team members; (3) explicit articulation of team member expectations, roles, and responsibilities; and (4) continuous communication across a complex landscape that encompasses scientific, regulatory, local communities, and commercial considerations (Sutton et al., 2019).

UC Davis has long recognized the importance of team science to timely discoveries that make a difference to the future of society and science itself. Interdisciplinary approaches have been a cornerstone of innovation. Interdisciplinary teams have been creating projects that, for example, positively affect the global environment, test the impact of climate change on the health of women agricultural workers, and measure the impact of technology on the future of agricultural farm work. STEM scientists in disciplines ranging from engineering, plant biology, and microbiology to the social sciences and health sciences together are creating programs that promote and facilitate team science. Multicultural perspectives are a natural fit with this endeavor and are influencing the next generation of STEM researchers in ways we are just beginning to appreciate.

Inter- and cross-disciplinary teams have integrated concepts, theories, methods, and multicultural perspectives into new research approaches that advance scientific innovation in ways that not only harness but also build upon increased specialization and knowledge fragmentation across diverse fields (Klein, 2010; Trochim et al., 2008). Growing evidence suggests that team science increases research productivity, helps to disseminate research findings across multiple disciplines (Vogel et al., 2014), and produces significant scientific outcomes as well as practical applications (Jordan, 2006). Team science also effectively engages and partners with local communities to generate knowledge critical for improving quality of life (Wallerstein et al., 2019).

\section{Lessons Learned and Best Practices}

The CAMPOS vision was to support discovery by establishing a research center that would change the face of STEM to reflect the multicultural diversity of California and beyond. The metrics of its successes included: (1) effecting institutional transformation with university leadership and National Science Foundation support, (2) adopting a mission and a vision, (3) designing a blueprint for action that can 
ensure that the best and the brightest receive appointments, retention, advancement, and (4) creating a sustainability plan for its future. Since its founding, CAMPOS has gained both state and national recognition for achieving excellence through diversity. It has become recognized as a national NSF model for recruitment, retention, and promotion of a diverse and inclusive STEM faculty with multicultural perspectives; it has also been recognized nationally by Forbes magazine as a destination place for women in science in STEM (Forbes, 2016). Women in science now wish to come to UC Davis not only to join its unique research community but especially, to help transform STEM science in the twenty-first century.

Despite fears among some STEM department faculty that standards might be lowered to increase diversity and inclusion, CAMPOS scholars' achievements at UC Davis to date include the following:

- Attaining National Science Foundation and National Institutes of Health grants, including one with a perfect NIH score with no scientific peer reviewer concerns or questions (a rare occurrence)

- Numerous peer-reviewed scientific publications

- National and international recognition for scientific discoveries

- Academy of Science Fellow selection

- Recognition of excellence through campus awards and recognition

- Promotion to tenure at UC Davis.

\section{Conclusion}

Recognizing the importance of diverse viewpoints in STEM is not new, but building and sustaining diverse, inclusive academic STEM environments is no easy task. It takes sustained resources, both intellectual and financial. Despite CAMPOS's numerous successes and achievements, sustainable institutional transformation is an ongoing process of transcending traditional and historical barriers. Optimizing the benefits, challenges, and opportunities of modern science and technology requires understanding and confronting existing and persistent social inequalities within the academy. Including multicultural perspectives in the discovery and creation of knowledge is needed to achieve diversity and inclusion in STEM fields; in turn, diversity and inclusion are needed if the United States is to remain a global leader in science.

At the University of California, Davis, the mission and vision that launched CAMPOS are springboards to a new beginning-one that fully embraces both our common humanity and the complexity of our shared environments. In its next phase, through the ongoing support of the provost and other campus leaders, CAMPOS is partnering with the arts and humanities to pioneer a new vision for joint discoveries that began with a multicultural perspective in science and will, in time, have broader social impact. 


\section{References}

Barker, J. A. (1992). Discovering the future: The power of vision. Infinity.

Barker, J. A. (Director). (1993). The power of vision video (motion picture). USA.

Bennett, L. M., \& Gadlin, H. (2012). Collaboration and team science: From theory to practice. Journal of Investigative Medicine, 60(5), 768-775.

Bergsieker, H. B., Wilmot, M. O., Cyr, E. N., \& Grey, C. B. (2020). A threat in the network: STEM women in less powerful network positions avoid integrating stereotypically feminine peers. Group Processes \& Intergroup Relations, 1368430219888274.

Bordas, J. (2012). Salsa, soul, and spirit. Berrett-Koehler.

Burgess, D. J., Joseph, A., Van Byr, M., \& Carnes, M. L. (2012). Does stereotype threat affect women in academic medicine? Academic Medicine, 87(4), 506-509.

Corneille, M., Lee, A., Allen, S., Cannady, J., \& Guess, A. (2019). Barriers to the advancement of women of color faculty in STEM: The need for promoting equity using an intersectional framework. Equality, Diversity and Inclusion: An International Journal, 38(3), 328-348.

Dutcher, E. G., \& Rodet, C. S. (2018, November 12). Which two heads are better than one?: Uncovering the positive effects of diversity in creative teams. From Social Science Research Network. https://ssrn.com/abstract=3283355

Farrell, L., \& McHugh, L. (2020). Exploring the relationship between implicit and explicit genderSTEM bias and behavior among STEM students using the Implicit Relational Assessment Procedure. Journal of Contextual Behavioral Science, 15, 142-152.

Flores, G., Mendoza, F. S., DeBaun, M. R., Fuentes-Afflick, E., Jones, V. F., Mendoza, J. A., \& Wang, C. J. (2019). Keys to academic success for under-represented minority young investigators: Recommendations from the Research in Academic Pediatrics Initiative on Diversity (RAPID) National Advisory Committee. International Journal for Equity in Health, 18(1), 93.

Forbes. (2016). The 13 Most Important STEM Colleges for Women, www.forbes.com nataliesportelli, March 29, 2016.

Freeman, S., Jr., Krier, K., Al-Asfour, A. A., \& Thacker, R. (2019). An examination of the barriers to leadership for faculty of color at US universities. Issues in Informing Science and Information Technology, 16, 361-376.

Jemison, M. (2019). The future never just happened; it was created. The National Academies of Sciences, Engineering, Medicine.

Jordan, G. (2006). Factors influencing advances in basic and applied research: Variations due to diversity in research profiles. In J. Hage, M. T. Meeus, \& C. Edquist (Eds.), Innovation, science, and institutional change (pp. 173-195). Oxford University Press.

Kantabutra, S. (2020). Toward an organizational theory of sustainability vision. Sustainability, 12(3), 1125 .

Klein, J. T. (2010). A taxonomy of interdisciplinarity. In R. Frodeman, J. T. Klein, C. J. Mitcham, \& J. B. Holbrook (Eds.), The oxford handbook of interdisciplinarity (pp. 15-30). Oxford University Press.

Kraimer, M. L., Greco, L., Seibert, S. E., \& Sargent, L. D. (2019). An investigation of academic career success: The new tempo of academic life. Academy of Management Learning \& Education, $18(2), 128-152$.

Maestre, F. T. (2019). Ten simple rules towards healthier research labs. PLOS Computational Biology. https://doi.org/10.1371/journal.pcbi.1006914

Miriti, M. N. (2020). The elephant in the room: Race and STEM diversity. BioScience.

Myers, I. B., \& Myers, P. B. (2010). Gifts differing: Understanding personality type. Davies-Black.

Pearce, J., Jensen, J., Valenti, D., \& Caffrey, J. (2020). A mathematical approach to the effects of gender bias and cross gender interactions on careers in STEM. Bulletin of the American Physical Society.

Ritchie, J. B. (1999). Crafting a value-driven vision for a national tourism treasure. Tourism Management, 20(3), 273-282. 
Sonnino, R. E. (2016). Health care leadership development and training: Progress and pitfalls. Journal of Healthcare Leadership, 8, 19-29.

Sutton, L., Berdan, L. G., Bolte, J., Califf, R. M., Ginsburg, G. S., Li, J. S., McCall, J., Moen, R., Myers, B. S., Rodriquez, V., \& Veldman, T. (2019). Facilitating translational team science: The project leader model. Journal of Clinical and Translational Science, 3(4), 140-146.

Szymanski, M., Fitzsimmons, S. R., \& Danis, W. M. (2019). Multicultural managers and competitive advantage: Evidence from elite football teams. International Business Review, 28(2), 305-315.

Toegel, G., \& Conger, J. A. (2003). 360-degree assessment: Time for reinvention. Academy of Management Learning \& Education, 2(3), 297-311.

Trochim, W. M., Marcus, S. E., Louise, M. C., Moser, R. P., \& Weld, P. C. (2008, March). The evaluation of large research initiatives: A participatory integrative mixed-methods approach. American Journal of Evaluation, 29(1), 8-28.

Vogel, A. L., Stipelman, B. A., Hall, K. L., Nebeling, L., Stokols, D., \& Spruijt-Metz, D. (2014). Pioneering the transdisciplinary team science approach: Lessons learned from National Cancer Institute grantees. Journal of Translational Medicine \& Epidemiology, 2(2).

Wallerstein, N., Calhoun, K., Eder, M., Kaplow, J., \& Wilkins, C. H. (2019). Engaging the community: Community-based participatory research and team science. In Strategies for team science success (pp. 123-134). Springer.

Xu, Y. J., \& Martin, C. L. (2011). Gender differences in STEM disciplines: From the aspects of informal professional networking and faculty career development. Gender Issues, $28(3), 1$.

Open Access This chapter is licensed under the terms of the Creative Commons Attribution 4.0 International License (http://creativecommons.org/licenses/by/4.0/), which permits use, sharing, adaptation, distribution and reproduction in any medium or format, as long as you give appropriate credit to the original author(s) and the source, provide a link to the Creative Commons license and indicate if changes were made.

The images or other third party material in this chapter are included in the chapter's Creative Commons license, unless indicated otherwise in a credit line to the material. If material is not included in the chapter's Creative Commons license and your intended use is not permitted by statutory regulation or exceeds the permitted use, you will need to obtain permission directly from the copyright holder.

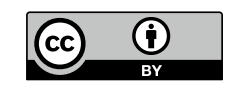

